\title{
Systems engineering education for inexperienced students by providing hand-on practices
}

\author{
Keiko Shimazu ${ }^{1, *}$, Yoshiaki Ohkami \\ ${ }^{1}$ Center of Education and Research of Symbiotic, Safe and Secure System Design, Keio University, Yokohama, Japan \\ ${ }^{2}$ Graduate School of System Design and Management, Keio University, Yokohama, Japan
}

\section{Email address:}

shimazu@sdm.keio.ac.jp (K. Shimazu), ohkami@sdm.keio.ac.jp (Y. Ohkami)

\section{To cite this article:}

Keiko Shimazu, Yoshiaki Ohkami. Systems Engineering Education for Inexperienced Students by Providing Hand-on Practices. Education Journal. Vol. 2, No. 3, 2013, pp. 64-71. doi: 10.11648/j.edu.20130203.11

\begin{abstract}
We developed a new hands-on type course for systems engineering. This is one of the courses at Keio University, Graduate school of System Design Management. The goal of the course is to provide the students with systems engineering methods proposed by INCOSE, one of the major organizations in the field. We report the effects of the course in this paper. There are two major contributions of the proposed course. The first is that we accept as many students from departments of social science as students from science and engineering. The second is that the course focuses on requirements development rather than on a certain technique or technology. These have not been realized in any of the previous engineering courses worldwide. We analyzed the effects of the course on the students and found out that the course helped them understand systems engineering in a short amount of time. Our students tried to make systems concept, architecture and design, and procured all items for implementing their systems, in order to solve the customer's demand; they want to maneuver an automatic cleaning machine from remote locations, even they are on their business trip.
\end{abstract}

Keywords: Systems Engineering; Education; Hand-on Exercise; Cleaning Robot

\section{Introduction}

We Japanese are in the midst of a national crisis. Even though there are many experts in various fields in this country, there still seems to be no clear solutions to the problems we are facing. Several years ago, Japan lost to Korea in an international bidding of a nuclear power plant in UAE. Japan is said to be lagging behind in the race for obtaining the rights to US railway construction. We believe that the reason for these situations is the lack of skills in systems engineering, not the capability of research and development within each field of expertise.

Systems engineering is defined as a technique of technology integration, which was developed in order to send men to the moon during the Apollo Program by utilizing the technology at that time. The mission of INCOSE (The International Council on Systems Engineering) is to develop the standard systems engineering methods, models and frameworks and spread them to the world. The systems engineering standards of INCOSE are based on ISO/IEC 12588 [1]. Best practice from IEEE, US Department of Defense, NASA, ECSS and various industries are also reflected in the standards. Some of the components, such as the Vee model, are deployed in Japan, but with different interpretations from those of INCOSE. Therefore the integration engineering, the essentials of systems engineering, has not yet become common. We believe that one of the reasons Japan, whose product manufacturing technologies and requirement development techniques are among the best in the world, cannot implement world-class systems is the lack of knowledge concerning systems engineering.

This paper presents a trial course in system engineering at Graduate School of System Design and Management (SDM). This trial course provides students with hand-on education for system design using commercially-off-the-shelf (COTS) components and sizable amount of development works within the limitation in one semester (15 classes, 30slots). In the latest semester that ended February 2010, we gave an assignment to build an automatic vacuum cleaner operated by a remote site, possibly from foreign countries. Almost all the hardware was prepared by the instructors. The students were required to design the total system and to develop a part of software program based on the specifications of the COTS. Systems engineering approach was emphasized for this including mechanical engineering and information technology as well. 
They started with defining Concept of Operations (ConOps) and requirement analysis, and then proceeded to systems architecting and design. After the functional requirements were defined, the physical realization and feasibility were checked with verificati4on and validation planning, and detailed design was performed, and finally coding, purchasing, and manufacturing of some parts. The components actually used to be 'iRobot' and small video camera as COTS and the use of 'Wi-Fi' networking was assumed.

This paper is organized as follows. We introduce the related works in Section 2 and our policy of the graduate school of SDM in Section 3. We describe the proposed course overview in Section 4 and the syllabus in Section 5. Section 6 presents the results from the course. We discuss the results in Section 7 and conclude the paper in Section 8.

\section{Related Works}

Research on hands-on type curricula applicable to various fields of engineering has been conducted by Bonnema et al, Castles et al and Yanfei et al [2, 3, 4]. However, they focus only on the methodology of manufacturing actual products and do not include concept development and requirement development processes of the projects. Schilling et al and Pomalaza-Raez et al has also reported on this type of classes $[5,6]$. These include the processes of concept development and requirement development, but are applied only in the field of aeronautics. We propose a novel hands-on type curriculum that includes both concept development process and the requirement development processes. Our curriculum can also be applied to education in various fields of the engineering domain.

Hole introduced the IBM case study on stakeholder requirement [7]. Interactive Solution Marketplace (ISM) in IBM is a single point of entry on the ibm.com website for browsing and searching for a suite of solutions as opposed to individual software and hardware items. ISM instructs the most important activity of systems engineering; stakeholder requirement. Consensus on core stakeholder requirements was achieved early during project development, and the technical reviews allowed the group to identify and resolve key issues before proceeding into subsequent phases.

Lande was interested in the maturation of master's students with backgrounds in mechanical engineering adjusting to a project-based learning experience centered on the design thinking methodology and processes [8]. He used a combination of theoretical approaches for design research, engineering education and the learning sciences.

At the University of California at Berkeley, embedded systems have become a traditional area of strength in the research agenda [9]. In parallel to this effort, a pattern of graduate and undergraduate courses has emerged that is the result of a distillation process of the research results. Sangiovanni-Vincentelli presented the considerations that are driving our curriculum development and we review our undergraduate and graduate program. In particular, we de- scribe in detail a graduate course (EECS249: Design of Embedded Systems: Modeling, Validation and Synthesis) that has been taught for six years. A common feature of our education agenda is the search for the fundamentals of embedded system science rather than embedded system design techniques, an approach that today is rather unique.

The three technical universities in the Netherlands (Eindhoven University of Technology, Delft University of Technology and University of Twente), abbreviated as 3TU, started a joint master on Embedded Systems in 2006 [10]. Embedded Systems is an interdisciplinary area of Electrical Engineering, Computer Science, Mechanical Engineering and Applied Mathematics. They studied the background of the master and presented the curriculum of the masters at the three sites.

The Plessey Telecommunications Company and Loughborough University disrupted the students' software development progress [11]. These actions appear mean and vindictive, and are labeled 'dirty tricks' in their words but their value was appreciated by both the students and their employers. The experiences and learning provided by twenty 'dirty tricks' are described and their contribution towards teaching essential workplace skills was identified. The feedback from both students and employers were mostly informal but the universally favorable comments received give strong indications that the courses achieved their aim of preparing the students for the workplace. They identify some limitations on the number and types of 'dirty tricks' that can be employed at a university and concludes that companies would benefit if such dirty tricks were employed in company graduate induction programmers as well as in university courses.

Forsberg et al. claim that education of systems engineering should be conducted with the involvement of the students from both the departments of social science and departments of science and engineering.

They also claim that systems engineering is not efficiently and effectively taught at universities due to the organizational structure. Departments and graduate schools are independently structured, and thus have independent curricula. They especially stress the drawbacks of separating MBA, which focuses on management, and engineering departments.

However, universities and graduate schools which claim to have adopted systems engineering from Europe and the US, courses of systems engineering practices can only be taken by students who have already taken courses from the engineering departments. There are a number of prerequisite courses, which also limits the students to those from the engineering departments.

\section{Outline of SDM Education}

\subsection{Objective and Basic Concept of the SDM Education}

We have developed a hands-on type course that considers the actual practice to improve these situations. 
To develop a hands-on type course in the master's program, we organized groups with both science and engineering students and social science students and had these groups solve the problems that we provided. This is an attempt which has not been conducted anywhere else in the world.

The objectives of the SDM are to foster strong leader for large scale projects and enterprises and creative system designer capable of planning, realizing and operating innovative systems and products, and reliable project manager creating new markets, satisfying consumer needs and operational value chain.

The basic concept of SDM is given as follows: System Design is a creative activity to bring a concept to a real being by balancing all the technical and social factors such as customer requirements, use objectives, essential functions, costs for R\&D and operations, adapt to the environmental changes, and trade-offs among stakeholders. System Management is to set up an adequate goal, to be achieved by balancing various factors including quality, cost, and schedule under risks and environmental changes.

System Design and Management should be a holistic approach by observing global trends of complex interactions among diverse languages, cultures and economics.

\subsection{Experiences and Issues}

For realization of the basic concept, we have set up the curriculum including courses to teach various methodologies of systems engineering, along the lines of Vee model (Fig.1.) It's a representative framework of systems engineering.

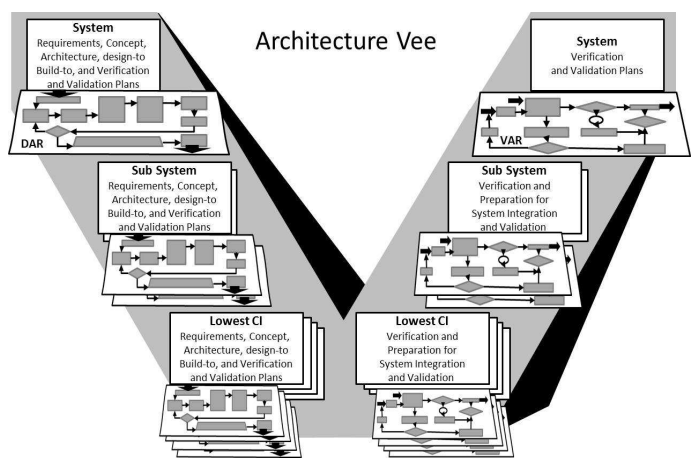

This is modified version of [12]

Figure 1. Various SE Methodologies Mapped on Vee.

When one year has passed since its establishment, however, it has turned out that students with no/little experiences in industries have a difficult time in understanding and appreciating the value of system engineering with various methods and tools. This is partly because about $20 \%$ of the students are fresh from undergraduate and partly because approximately $30 \%$ of the students have very little experiences in engineering. Without such experiences, it is practically impossible to understand meanings and importance of requirement definition phase and architecture de- sign phase that is critical to systems engineering process. This has motivated a hand-on practice.

\section{Course Overview}

\subsection{Course Schedule and Length}

The course is held in both the spring and autumn semester. The two courses have the identical contents. Systems engineering is a practical science. With more experience, knowledge also increases. We recommend the students take the proposed hands-on type course more than once even though the syllabi are identical.

Each class consists of two consecutive slots, each slot lasting 90 minutes. The scale of the product to be created should be completed in 15 days (15 classes, 30 slots), each slot is 90 minutes, as mentioned above.

This is due to the fact that the groups consist of both engineering students and social science students. Since the engineering students have the implementation skills, we were worried that the homework may become a burden to the engineering students. The first step in the 1 st slot is formulated groups and each has 4,5 , or 6 students.

\subsection{Course: Solution Goal}

Student groups take an Initial requirement document, in which there is the main goal of the system. Customers want to maneuver an automatic cleaning machine, iRobot (Fig 2), from remote locations even they are on their business trip.

Also a set of COTS provided by the instructors to each group can be combined in several ways. This class is not about reaching the only correct goal. As in the real systems engineering world, we prepare several methods to achieve the goal, and have the students go through selection criteria from multiple viewpoints. This class offers the experience of studying the really, finding the optimal solution and executing the processes of systems engineering to achieve the solution.

Students should achieve an acceptance review of the supposed clients as assigned to other instructors, at the final class.

\subsection{Course: Implementation Structure}

\subsubsection{Lecturer}

\section{3 lecturers.}

1 conducts the lectures and leads the classes, 2 act as the pseudo board member and manager of sponsored company.

\subsubsection{Teaching Assistant}

2 second year master students. Students who received good marks in the previous course supports.

Students

Students in either Master's or Doctor's program at the Graduate School of System Design and Management at Keio University. 1 group consists of 4 to 6 students. Each group has students from engineering and students from social science. 
Emphasis is placed not acquiring skills to develop hardware/software components based on conventional engineering techniques, but on the skills of completing a system by applying the standard approach of systems engineer under the constraints of schedule and human resources.

\subsection{Course: Materials}

\subsubsection{Main Requirement Presented by the SPONSOR}

Using iRobot, an automatic vacuum cleaner as COTS, groups are asked to implement a system in which the users are able to clean the rooms at home from abroad. This is a new product from the sponsor corporation mentioned above, and is designated as a prototype.

\subsubsection{Textbook}

We use the book named Visualizing Project Management [12] as the main textbook. INCOSE systems engineering handbook [13] is introduced as a reference, and if necessary, we also refer to PMBOK guide [14].

\subsubsection{Provision of Lowest Configuration Items}

We have prepared hardware items, called the Lowest Configuration Items (LCI) beforehand in view of schedule constraint to establish a system within the 30 slots. Specifically, the instructors used COTS as LCI. For this preparation, the instructors have assumed several patterns of ConOps from the Initial requirements and devise a systems architecture which would achieve each of the ConOps. Furthermore, the instructors prepared the LCI that would be required to establish all these system architectures before the course started. Therefore, some of the COTS that the instructors prepared may not be needed depending on the ConOps and the systems architecture that the students select.

The 3 instructors were in charge of the hands-on exercise: A professor as a customer; an associate professor as a consultant on information and communication engineering; an associate professor as a consultant in systems engineering.

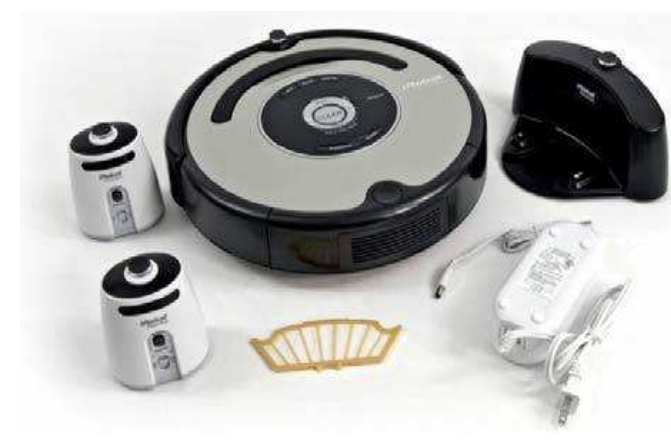

Figure 2. Automatic Cleaning Machine 'iRobot' as COTS.

\section{Our Syllabus}

In the proposed course, we defined project period as Concept Stage and Development Stage in the standard system Lifecycle from ISO/IEC 15288. Following the proposal by Forsberg et al. Study Period, or Concept Stage, consists of 4 phases; User Requirement Definition Phase, Concept Definition, System Specification, Acquisition Preparation, and Implementation Period, or Development Stage consists of 3 phases; Source Selection, Development, Verification. In this course, we follow these project Lifecycle phases in order [15].

\subsection{Class1 : Orientation}

Explanation of course syllabus and overview of systems engineering. Students are asked to provide a self evaluation on each of the systems engineering terms based on how well they can explain the term to a third person on the scale of 5. Proposals concerning the implementation method of the remotely controlled vacuum cleaning system are handed in. Students are also asked to write down the major of the undergraduate degree.

\subsection{Class2 : Understanding the Importance of Require- ments Development}

We explain that the essence of systems engineering is to satisfy the requirements of stakeholders, and have the students practice concretizing (converting into engineering and quantitative terms) vague requirements. All the processes from this class on are conducted in groups. We explain the role of decision gate and the implementation method in this course.

\subsection{Class3 : User Requirement Analysis Phase}

Each group receives the identical "system concept plan" as initial requirements from the pseudo sponsor corporation and acquires the skill inventory of group members and approximate budget information. Groups are asked to improve the initial requirement documents and separate the text into those related to implementation method and those related to state change to achieve by the introduction of the system.

\subsection{Class4 : User Requirement Definition Phase}

The groups list up the possible stakeholders and identify the most important requirements from each stakeholder viewpoint. After the lecture on the basic structure of documents, the groups try to find inconsistency in the requirements and update the required documents with the sponsor corporation.

\subsection{Class5 : User Requirement Development Phase}

Groups develop ConOps and prepare multiple system implementation plans. They also conduct the decision gate to agree upon the basic requirements.

\subsection{Class6 : Concept Definition Phase}

Groups select few candidates for the implementation method concepts and adjust the balance between the expenses for the purchase of necessary components and the 
advantages of the implemented functions.

\subsection{Class7 : System Specification Phase}

Student groups select the implementation method concept, prepare the systems architecture and check the feasibility using models and simulations.

\subsection{Class8 : System Components Specification Phase}

Student groups select each of the components and implementation method of the interface. By adjusting the balance of the expenses, grapes reach an agreement with the sponsor corporation on ConOps and the decision gate.

\subsection{Class9 : Acquisition Planning Phase}

Student groups identify who collects/produces/assembles what at what period of time, and prepare a schedule. The groups must focus on the specifications of verification and validation and must agree with the sponsor corporation on the decision gate.

\subsection{Class10 : Source Selection Phase}

Student groups decide on where to buy the necessary components from and order them to fix the final amount of expense. The groups start the integration with the parts that they received.

\subsection{Class11-13 : Development phase and Verification Phase}

Student groups conduct system integration and verification according to the schedule.

\subsection{Class14 : Preps for Final Decision Gate}

Student groups conduct validation and make final adjustments to each of the documents.

\subsection{Class15 : Final Decision Review (Acceptance Re- view)}

Student groups make the final presentation and demonstration to the sponsor corporation. The sponsor corporation tests the system and groups receive the final approval.

\section{Results of Exercise}

\subsection{Examples of Developed System}

We will present some characteristic output from each of the semesters.

(1) Autumn 2009

Members: a Dutch student from Delft University of Technology, a French student in Master's program, a Korean-American student in Master's program, an American student in Master's program and a Japanese student in the Master's program. Their majors: management, mechanical engineering, management, financial engineering, physics (in order).
System outline

The goal is to let the users enjoy the service daily without the knowledge of information technology or mechanisms. The web-service system is implemented based on Wi-Fi. The web camera operation is also conducted on the servers.

(2) Spring 2010

4 auditing students from the graduate school of Aerospace engineering at Nihon University and other universities.

System outline

To utilize the skills of the members, they designed a hardware based system. By calling the number for the automatic vacuum cleaner from abroad using a cell phone, the device above the remote controller activates. The sound of the different number buttons drops corresponding bars of the device to onto the remote controller, which in turn operates the remote controller buttons.

(3) Autumn 2010

A Chinese student from Delft University of Engineering, a Japanese-American student in the Master's program, a Korean-American student in the Master's program. Their majors: electronic engineering, management, public policy (in order).

System outline

The PC inside the room is remotely accessed using PC and PDA via the Internet. The PC in the room operates the vacuum cleaner using Bluetooth. Validation was conducted from 4 different countries.

(4) Spring 2011

4 Japanese students from Master's program. Their majors: commerce, aerospace engineering and mathematics and informatics, electronic engineering, and law. 3 of them are adult students.

System outline

The vacuum robot and the user communicate through twitter to control the robot. Communication is conducted between PC or PDA and the PC in the room. The indoor PC sends a command from twitter to the robot using Bluetooth. They also focused on the fact that it is hard to monitor the movement of the cleaning robot through web camera when there is a time difference between the user's location and the room. By tracking the record through twitter, users can control the robot as if playing a game(Fig. 3).

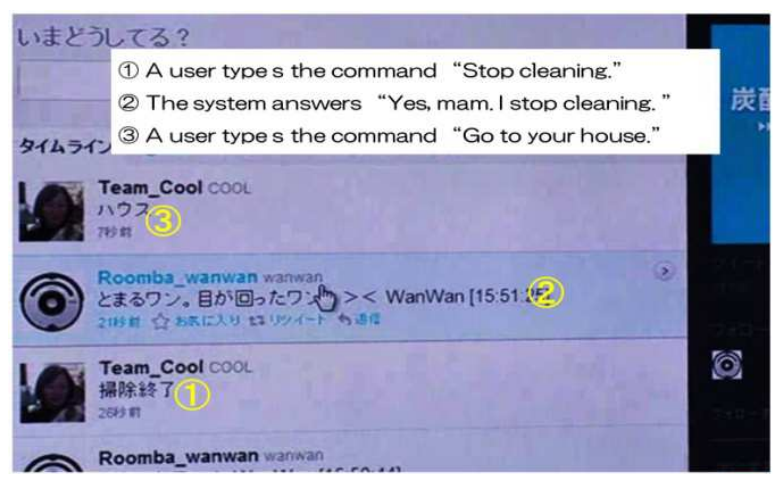




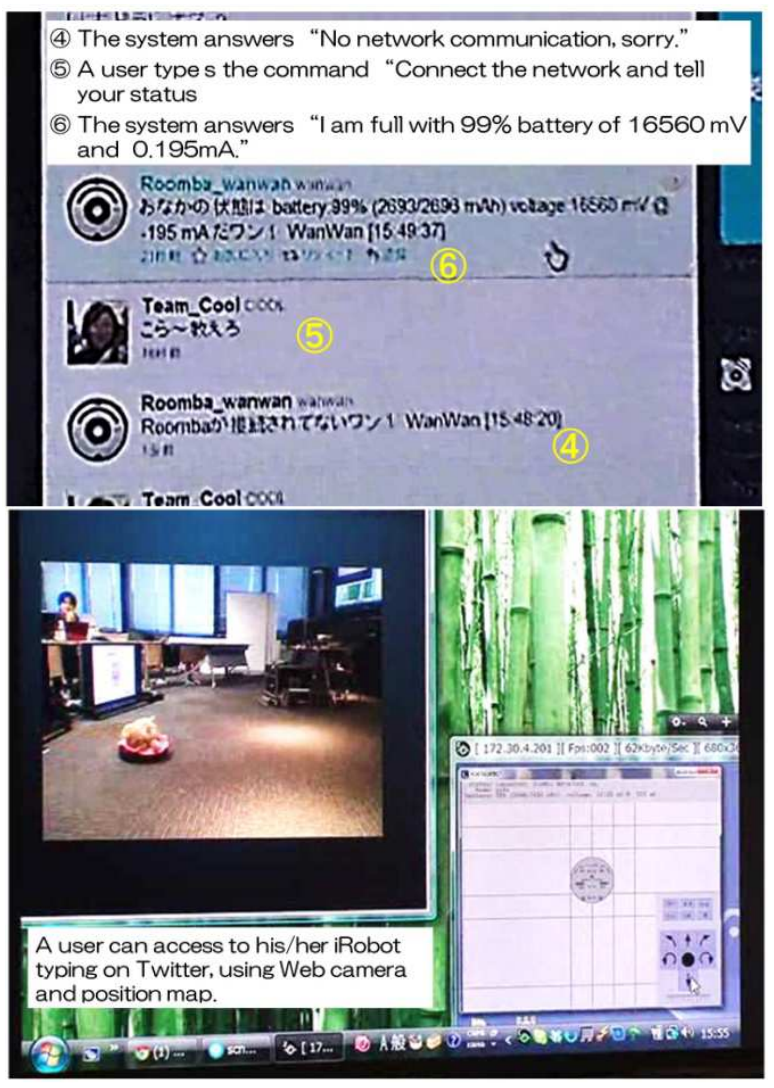

Figure 3. Example of Students' Output.

\subsection{Observing the Students' Understanding of Systems Engineering}

We asked all the students concerning their understanding of 10 important systems engineering terms, as table 1 . This has been conducted in the first and the final class. In the first and the last class, students recorded the understanding of each term. The understanding is on a scale of $5 ; 1$ indicates that students know the term and 5 indicates that they can explain it to a third person. Fig. 4 presents the results in a radar chart. The evaluation is higher in the last class in all of the semesters. We also can see that the evaluation of the initial class is higher in the autumn semester. In spring 2010, the evaluation of WBS and schedule for the final class is lower compared to other semesters.

Table 1. SE term understanding self-evaluation results before and after the hands-on type course.

\begin{tabular}{|r|l|}
\hline \multicolumn{2}{|c|}{ Selected Systems Enginering Terms } \\
\hline 1 & System \& Systems Engineering \\
\hline 2 & Vee model \& System Lifecycle model \\
\hline 3 & Project Scope/ Boundary \\
\hline 4 & Stakeholder Requirement \& Systems Requirement \\
\hline 5 & Requirement Development \\
\hline 6 & WBS \& Schedule \\
\hline 7 & Decision Gate \\
\hline 8 & ConOps \\
\hline 9 & Architecture \& Systems Design \\
\hline 10 & Verification \& Variation \\
\hline
\end{tabular}

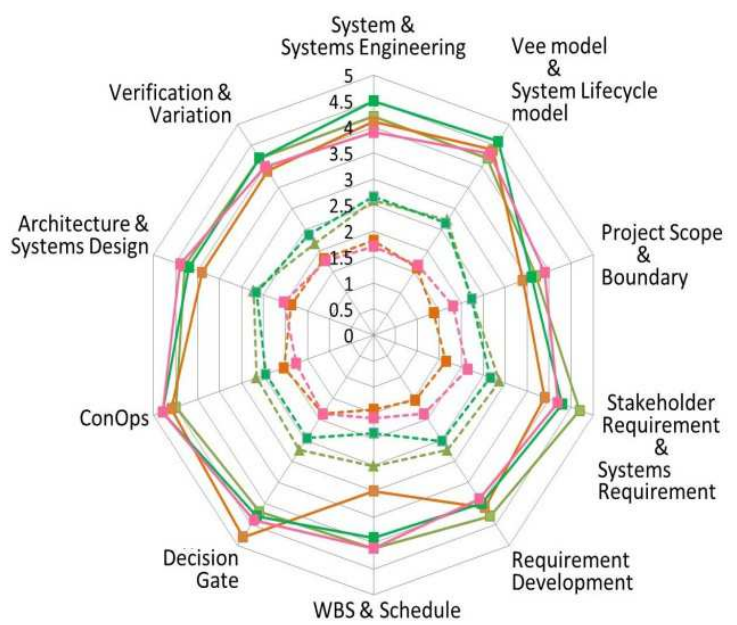

\footnotetext{
- 2009 Evaluation on the 1st slot of Autumn Semester

-2009 Evaluation on the last slot of Autumn Semester

- - 2010 Evaluation on the 1st slot of Spring Semester

-2010 Evaluation on the last slot of Spring Semester

- 믈-2010 Evaluation on the 1st slot of Autumn Semester

-2010 Evaluation on the last slot of Autumn Semester

- -2011 Evaluation on the 1st slot of Spring Semester

-2011 Evaluation on the last slot of Spring Semester
}

Figure 4. SE term understanding self-evaluation results before and after the hands-on type course.

\section{Discussion and Lessons Learned}

In all of the terms, we have succeeded in enhancing the knowledge students concerning systems engineering from INCOSE through the proposed course. The difference of self evaluation in the first class in the spring semester and the autumn semester is due to the fact that the students that participate in the autumn semester are from overseas or graduates of European or American universities. In Europe and America (and even in Asia, mainly Singapore), education in INCOSE systems engineering is widely available, and the fact that students from these regions have some basic knowledge seems to have affected the evaluation results. For the spring semester of 2010, the scores of WBS and schedule seem to be lower than those of other semesters because we could not allocate enough time to these elements. In the proposed hands-on type course, we tried not to avoid homework as much as possible and have students finish the processes within a class (Section 3.1). The lecturer adjusted the difficulty of the contents and the amount of explanation at the start of the course according to understanding of students and the progress of operations. In this semester, we allocated more time for understanding the requirements from the requests of students. We had to decrease the time spent on WBS and schedule. This, as a result, led to the difference in the evaluation.

As explained above, our hands-on course has turned out to be useful to draw potential capability of the students and also to educate them the group working and leadership. However, one of the points all of them have missed is related to the 
following simple question: "In what state the system is: active, sleeping or completely off?" and "How can you switch on and off?"

We have earned many lessons learned and we are planning to reflect these lessons on the next classes with the following items to be taught to the students in addition to the curriculum as explained in the previous sections.

Collecting information on the COTS from the suppliers and from WEB site

Familiarization to the hardware equipments, especially the cleaner, iRobot, and video-camera

Familiarization to the COTS operations by investigating the manuals in detail

Configuration management especially for documentation

Consideration of guarantees and maintenance schemes

ConOps of the total system

The hands-on class intentionally had students' experiential lessons learned. In other words, we do not present methods to avoid mistakes in advance. After presenting standard systems engineering processes, we let the students work with their own ideas, and when the work comes to a halt due to some problems, the instructors will then lecture the students. To be precise, we helped the students clarify the causes of the problems and gave a brief guide on what requires rework. The cause of the first rework was the lack of check on the feasibility of component integration, and the instructors assumed several integration methods and combination methods and instructed the students to select the optimal one. The second rework was due to the incompleteness of the initial requirements. Specifically, failing to limit the functional domain of remote operations ceased functioning enhancement operations on the vacuum cleaner, which is one of COTS products. The instructors told the students to sequentially conduct boundary refinement at each process of systems engineering, and students succeeded in function identification.

The lesson that instructors learned through these two rework processes is that the students who have no practical experience tend to actively propose free and intuitive implementation methods from (parts of) requirements in hands-on classes. For the ones who have practical experience have the tendency to be trapped within their experiences and avoid techniques and methods they have no experience with, there is a possibility of revolutionary breakthroughs. On the contrary, there is also a tendency that the students cannot look at other implementation methods and rush to the fabrication process without much consideration. This was another lesson learned. In real life systems engineering, we assume several implementation methods to achieve risk dispersion and process according to schedule, and select an optimal method from multiple viewpoints. Therefore, cutting-edge technologies, in many cases, are not adopted (due to insufficient feasibility). Students, who have no practical experience, tend to assume cutting edge technologies and methodologies as the only implementation method due to personal interest (in most cases of intellectual interest, which is excellent) and conduct the processes, which in turn cause rework processes.

\section{Concluding Remarks}

We started a hands-on course at a newly established graduate school of Systems Design and Management at Keio University, the oldest university with 150 years of history in Japan. To improve the situation in Japan where universities hardly provide lectures on the essence of the systems engineering methodology, we aim to establish a framework of university education which fosters experts that can handle embedded system development. Through this course with hands-on exercise, we have recognized tendency in student attitudes towards focusing on specific methods and tools, and moving straight into implementation without sufficient consideration in previous processes such as requirement development and system architecting. We have decided to continue this course after modifying the contents by reflecting the lessons learned.

We will monitor the individual changes of students to develop a more efficient and effective course and prepare a similar course for corporations as future work.

\section{Acknowledgements}

This work was supported in part by Grant in Aid for the Global Center of Excellence Program for "Center for Education and Research of Symbiotic, Safe and Secure System Design" from the Ministry of Education, Culture, Sport, and Technology in Japan.

My heartfelt appreciation goes to Ms. Terumi Fukuhara whose help was of inestimable value for our study.

\section{References}

[1] IEEE Computer Society, ISO/IEC 15288:2008(E) IEEE Std $15288^{\mathrm{TM}}-2008$ (Revision of IEEE Std 15288-2004), "Systems and software engineering — System life cycle processes"

[2] G.M. Bonnema, I.F. Lutters-Weustink, and F.J.A.M van Houten, "Introducing systems engineering to industrial design engineering students with hands-on experience," 2005. ICSEng 2005. $18^{\text {th }}$ International Conference on Systems Engineering, pp.408-413, August 2005

[3] E.A. Gonzalez, M.C.G. Leonor, P.A.T .Mangulabnan, J.J.S.L.C. Kau, and M.W.U. Reyes, "Work in progress _ an educational tool for teaching linear and control systems," 2007. FIE '07. 37th Annual Frontiers In Education Conference - Global Engineering: Knowledge Without Borders, Opportunities Without Passports, pp. T3J-14-T3J-15, October 2007

[4] R. Castles, V.K. Lohani, and P. Kachroo, "Utilizing hands-on learning to facilitate progression through Bloom's taxonomy within the first semester," 2009.FIE '09. 39th IEEE Frontiers in Education Conference, pp.1-5, October 2009

[5] Liu Yanfei, and C. Pomalaza-Raez, "Concept learning embedded in a freshman engineering project on energy sca- 
venging," 2010 International Conference on E-Health Networking, Digital Ecosystems and Technologies (EDT), pp. 360-363, April 2010

[6] K. Schilling, "Design of pico-satellites for education in systems engineering," IEEE Aerospace and Electronic Systems Magazine, vol.21, pp. S_9- S_14, July 2006

[7] K. Forsberg, H. Mooz, and H. Cottenerman, "Visualizing Project Management," Charts and Frameworks for Mastering Complex systems, Wiley, 3rd edition, pp.xxi-xxvi, 2005

[8] Ibid., pp.16-17

[9] Ibid., pp.26-27

[10] C. Haskins, K. Forsberg, M. Krueger, D. Walden, and R. Hamelin, "SYSTEMS ENGINEERING HANDBOOK, A GUIDE FOR SYSTEM LIFE CYCLE PROCESSES AND ACTIVITIES," INCOSE-TP-2003-002-03.2.1, pp. v-vi , 2011
[11] K. Forsberg, H. Mooz, and H. Cottenerman, "Visualizing Project Management," Charts and Frameworks for Mastering Complex systems, Wiley, 3rd edition, 2005

[12] Ibid., pp.148

[13] C. Haskins, K. Forsberg, M. Krueger, D. Walden, and R. Hamelin, "SYSTEMS ENGINEERING HANDBOOK, A GUIDE FOR SYSTEM LIFE CYCLE PROCESSES AND ACTIVITIES," INCOSE-TP-2003-002-03.2.1, 2011

[14] Project Management Institute, A Guide to the Project Management Body of Knowledge, 4th Edition, 2008

[15] K. Forsberg, H. Mooz, and H. Cottenerman, "Visualizing Project Management," Charts and Frameworks for Mastering Complex systems, Wiley, 3rd edition, pp.84-102, 2005 